Document downloaded from:

http://hdl.handle.net/10251/37944

This paper must be cited as:

Luis Humberto BERRIO; Sales Maicas, S.; Barrera Vilar, D. (2012). Bend Sensor Using Fiber Bragg Gratings to Determinate the Distance from the Sensor to the Neutral Axis in Compression and Extension. IEEE Latin America Transactions. 10(5):2040-2044. doi:10.1109/TLA.2012.6362346.

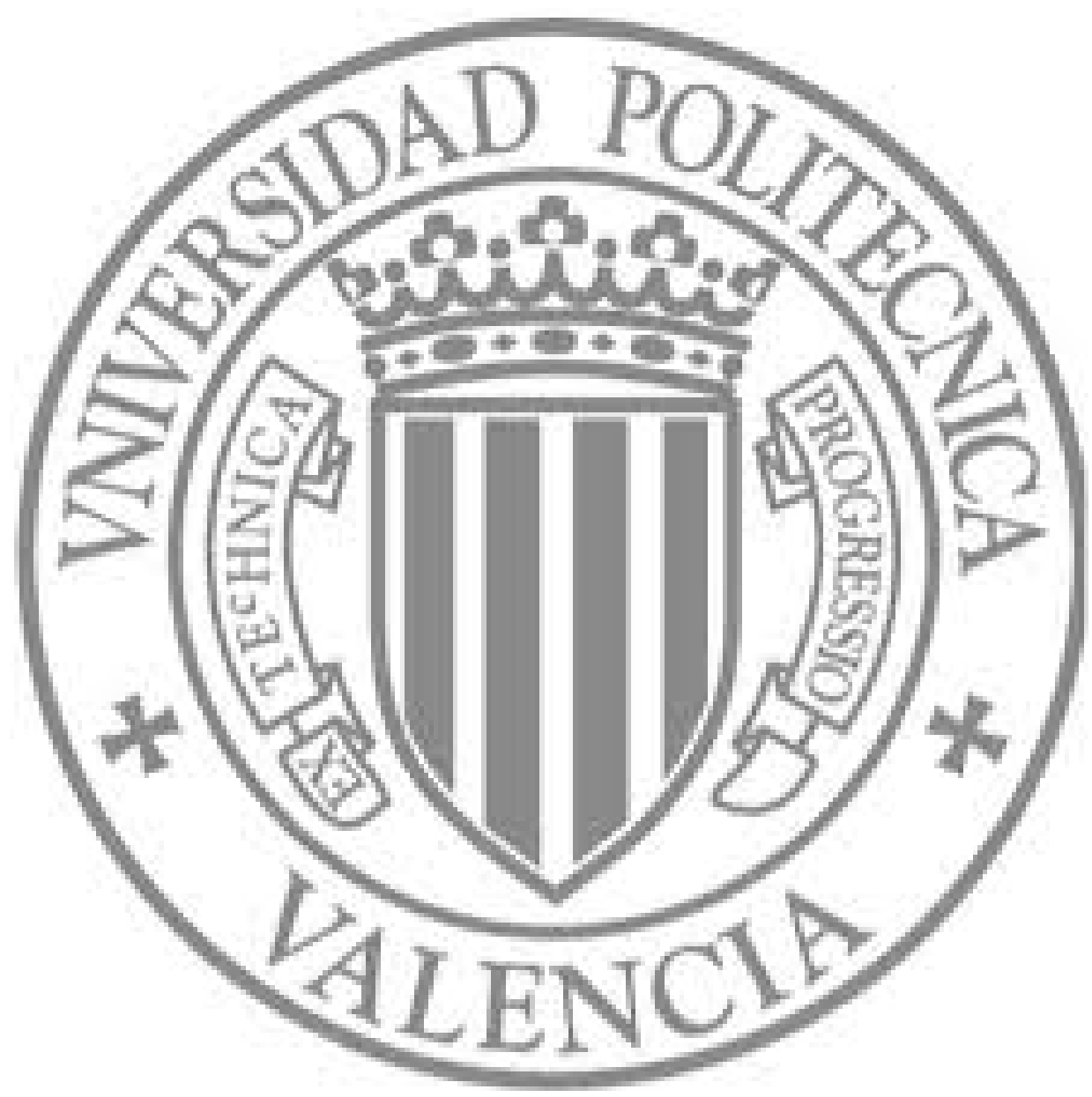

The final publication is available at

http://dx.doi.org/10.1109/TLA.2012.6362346

Copyright Institute of Electrical and Electronics Engineers (IEEE) 


\title{
Bend Sensor Using Fiber Bragg Gratings to Determinate the Distance from the Sensor to the Neutral Axis in Compression and Extension
}

\author{
L. H. Berrío, S. Sales, D. Barrera
}

\begin{abstract}
The following paper presents a bend sensor that consists of three Bragg gratings inside a standard fiber optic for the characterization of the reflected wavelengths with respect to the radius of curvature in conditions of compression and extension, and it is used to find the distance between the bend sensor and the neutral axis. Actually, there are many problems with the package and the union techniques of the bend sensor and the surface that will be sensed. Knowing this distance it is possible to recognize the behavior in compression and extension of a unique way, because for different sensor positions with respect the neutral axis and with a same value of radius of curvature, there will be different reflected wavelengths, but it will be possible to find the real radius of curvature using different distances.
\end{abstract}

Index Terms-FBG, compression, extension, bend sensor, radius of curvature, neutral axis, strain.

\section{INTRODUCCIÓN}

$\mathbf{L}$ A fibra óptica ha permitido el desarrollo y diseño de nuevos sensores ópticos, que en muchas situaciones son mejores que los diseños electrónicos, debido a características como la inmunidad a interferencias electromagnéticas y su tamaño reducido [1]. Por ello, el estudio y análisis de estos sensores se ha venido incrementando a lo largo de los años.

Entre los grandes desafíos a los que se enfrenta la ingeniería contemporánea están el mantenimiento y la seguridad en el funcionamiento de infraestructuras civiles. Las técnicas y métodos de monitorización estructural permiten identificar la presencia, ubicación y magnitud de daños a partir de cambios en sus características estáticas y dinámicas, así como analizar la seguridad y fiabilidad de las construcciones civiles en base a las variables físicas medidas. La incorporación de sensores en las infraestructuras civiles permite realizar labores de control y diagnóstico de posibles problemas, para luego proponer métodos para al mantenimiento y reparación de la infraestructura antes de un deterioro irreversible [2]. Las magnitudes a determinar en infraestructuras civiles suelen ser las relacionadas con deformaciones, vibraciones y temperatura, entre otras. En la actualidad se pueden encontrar nuevos dispositivos basados en fibra óptica para la medida precisa de este tipo de magnitudes [3].

L. H. Berrío, Universidad de Antioquia (UdeA), Medellín, Colombia, berrio77@yahoo.com

S. Sales, Universitat Politècnica de València (UPV), Valencia, España, ssales@dcom.upv.es

D. Barrera, Universitat Politècnica de València (UPV), Valencia, España, dabarvi@iteam.upv.es
Algunos de estos sensores utilizan redes de difracción de Bragg, que son pequeños tramos, de pocos centímetros de longitud, donde se ha modificado el índice del núcleo de forma periódica [4]. Esta variación permite que se refleje una parte del espectro de la señal incidente y deja que el resto pase a través de ésta. Se puede definir la longitud de onda central de la banda reflejada, $\lambda_{\text {Bragg }}$, como un valor proporcional al producto [5] de la periodicidad de la variación, $\Lambda$, y el índice de refracción efectivo de la fibra, $\eta_{e f f}$, como se muestra a continuación:

$$
\lambda_{\text {Bragg }}=\Lambda \eta_{\text {eff }}
$$

Con base en este principio, se pueden lograr bandas de longitudes de onda reflejadas lo más estrechas que sea posible, para que los cálculos desarrollados se basen en esta frecuencia central [6].

El uso de las redes de difracción de Bragg (FBGs) para medir deformaciones tiene ventajas como sus pequeñas dimensiones, fácil fabricación y economía. Uno de los problemas que se afronta es que dependiendo del tipo de empaquetado o la forma en que se adhiere la FBG a la estructura a sensar, se obtienen diferentes valores y respuestas [7]. Para caracterizar adecuadamente una medición, se tiene en cuenta la distancia entre la ubicación real del sensor respecto al eje neutro de deformación, independiente del empaquetado o técnica de adhesión.

Las respuestas de las FBGs cambian debido a condiciones mecánicas, expansiones térmicas y entornos termo-ópticos y fotoelásticos. Diseños novedosos de empaquetados de sensores con redes de Bragg permiten distinguir entre las medidas obtenidas por deformación y por temperatura [8], además de servir como protección. Para llevar a cabo estas mediciones simultáneamente, se requiere variar el peso y el ancho del empaquetado del sensor, y así se evita el efecto que genera una variable sobre la otra. Estos nuevos encapsulados también afectan la distancia del sensor al eje neutro de curvatura, afectando la respuesta en frecuencia para un radio de curvatura dado. La necesidad de usar empaquetado radica en que los sensores ópticos, como los que hacen uso de las FBGs, están más propensos a daños mecánicos cuando no lo tienen y este deterioro de la fibra genera deficiencia en la toma de mediciones. 


\section{DISEÑO DEL SENSOR DE CURVATURA}

\section{A. FBGs en la fibra}

En los experimentos realizados en el curso de esta investigación, se fabricaron tres FBGs consecutivas en una misma fibra estándar con el fin de obtener la relación que conduzca a encontrar el valor de la distancia entre el sensor y el eje neutro de curvatura. En la fig. 1 se muestran las dimensiones de las FBGs dentro de la fibra. Estas FBGs se diseñaron de tal forma que pudiesen reflejar un rango muy estrecho de longitudes de onda ubicado en la tercera ventana $(\sim 1550 \mathrm{~nm})$, pero con una pequeña diferencia en la frecuencia reflejada por cada una, para que al momento de las mediciones no haya un solape entre las señales reflejadas.

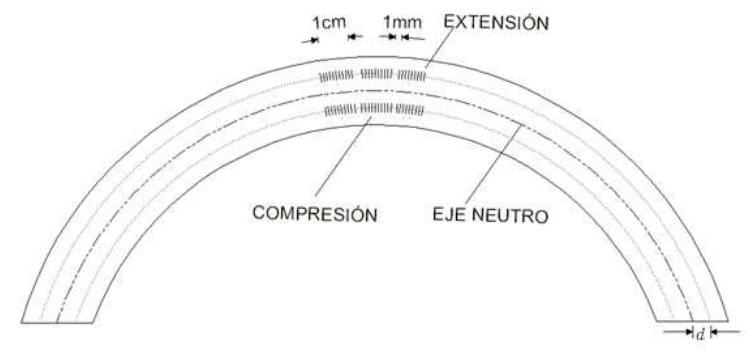

Fig. 1. Fibra óptica estándar con las FBGs en su interior.

El eje que pasa a través del centroide de una sección de una estructura civil, llamado eje neutro o eje centroidal, tiene fuerzas a tracción a un lado del eje, mientras que sobre el otro son fuerzas a compresión, lo cual permite decir que la resultante de las fuerzas sobre el eje neutro es cero [9]. Cuando se adhiere una red de Bragg sobre una superficie, el eje neutro de la estructura completa varía, y otros factores como el empaquetado también modifican su posición final. En pocas palabras, esta es la región que no se comprime ni se extiende, por lo que su variación de deformación $(\Delta \epsilon)$ será siempre cero, sin importar el radio de curvatura de la fibra [10].

\section{B. Montaje mecánico del sistema}

Para llevar a cabo las mediciones de compresión y de extensión, se usó una lámina delgada de aluminio de $0,4 \mathrm{~mm}$ de espesor. El sensor constituido por las tres FBGs se adhirió a la parte superior de la lámina usando una capa muy fina de resina. Con el fin de poder realizar las mediciones de compresión y extensión al mismo tiempo, una segunda fibra con las mismas características de la primera se colocó en la parte inferior. En la fig. 2 se muestra el montaje mecánico del sistema, y se indica la fibra que se comprimirá y la fibra que se expandirá en términos de longitud de onda. La sección de circunferencia mostrada en la fig. 2 representa la lámina con las dos fibras, una de las cuales está en compresión y la otra en extensión, como se observa en la fig. 1.

\section{Adquisición de datos}

Se utilizó una fuente de luz blanca, con un espectro uniforme en la región de la tercera ventana, para que las FBGs

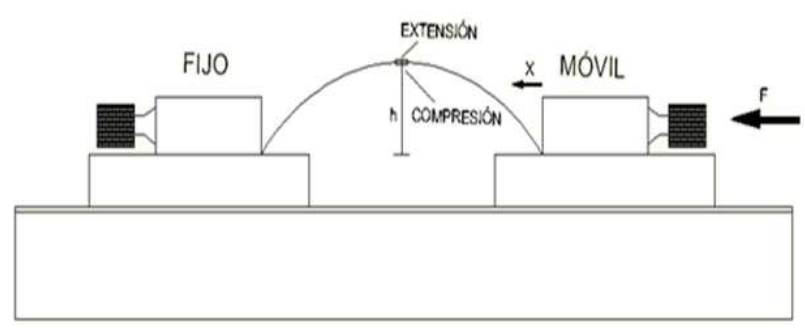

Fig. 2. Montaje mecánico del sistema

pudiesen reflejar una longitud de onda en particular. La señal proveniente de la fuente blanca se hace pasar por un circulador, el cual tiene conectada la fibra con las FBGs en uno de sus puertos [11]. Las bandas estrechas de longitudes de onda que son reflejadas por las tres FBGs pasan de nuevo por el circulador [12], que conduce dichas señales sobre otro puerto de salida que está conectado a un analizador de espectro óptico, OSA. Con el esquema planteado, las respuestas de las tres FBGs se monitorizan simultáneamente. La fig. 3 muestra la conexión de los elementos ópticos.

Para la medición de la longitud de onda reflejada, se realizaron desplazamientos horizontales variables sobre un extremo de la lámina, por medio de una prensa mecánica [13] y los desplazamientos en la altura de la lámina eran cada $2 \mathrm{~mm}$. Se logró un barrido de 21 muestras por FBG en el experimento. En cada medición se calculó la longitud de onda y el radio de curvatura.

En el analizador de espectros, los seis picos de longitud de onda se ubican en la tercera ventana, pero distanciados entre sí. Tres de ellos disminuyen en longitud de onda a medida que la altura aumenta, mientras que los otros tres aumentan en longitud de onda a medida que la altura disminuye.

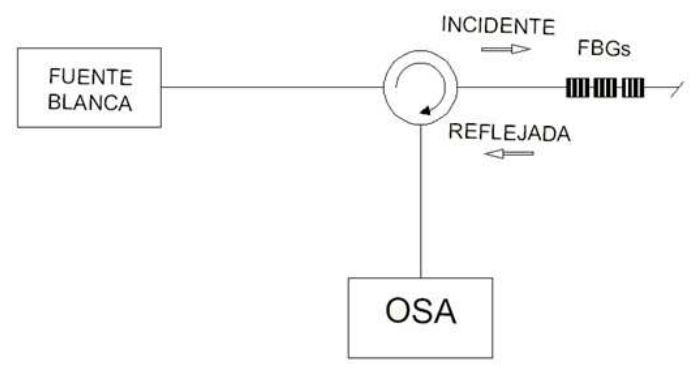

Fig. 3. Esquema de conexión de los elementos ópticos para la adquisición de datos.

\section{Curvas de sensado}

Con la captura de los valores pico en cada banda de longitud de onda para las FBGs, se obtienen gráficas de longitud de onda respecto al radio de curvatura, tanto en compresión como en extensión. El radio de curvatura se define como el inverso del radio de la circunferencia que describe la lámina de aluminio a lo largo de su eje neutro. Existen otras topologías sobre vigas en voladizo sobre las que hay grandes desarrollos en temas de sensibilidad de los sensores con FBGs y de medidas simultáneas de temperatura y deformación [14], 
[15],[16]. Este análisis se concentró sobre estructuras que tienen puntos de apoyo en sus dos extremos.

En la fig. 4 se muestra cómo se determina el radio de curvatura geométricamente, donde la altura es el valor conocido y es medido físicamente [17]. Para efectos de la presente investigación, se usó únicamente la zona en la que es posible modelar la deformación usando la ecuación de la circunferencia. Esta relación será válida hasta que la altura sea como máximo el doble de la longitud horizontal donde se curva la lámina, ya que a partir de allí no se tendrá un radio de circunferencia y se deberá tener en cuenta la deformación que ésta va sufriendo, además la pérdida de potencia de la señal óptica será mayor.

Si se aplica el teorema de Pitágoras al triángulo $\overline{O B C}$ se obtiene la siguiente ecuación:

$$
(r-h)^{2}+\left(\frac{F}{2}\right)^{2}=r^{2}
$$

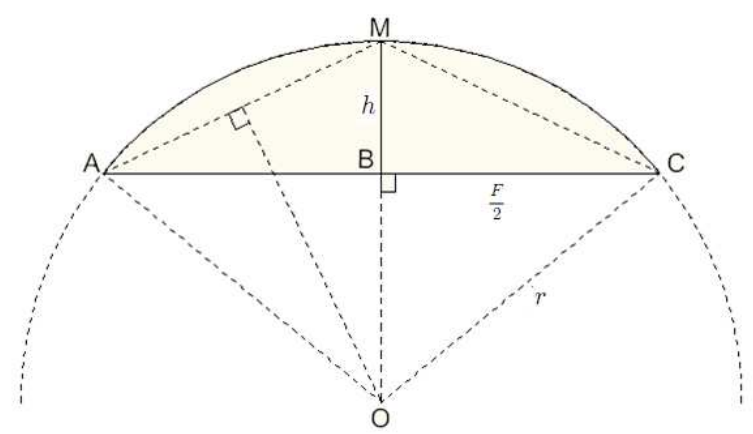

Fig. 4. Relación geométrica para el cálculo del radio de curvatura.

En (2), $r$ es el radio de la circunferencia, $F$ es la longitud del segmento de recta $\overline{A C}$, el perímetro de la sección de circunferencia $\overline{A M C}$ describe la longitud de la lámina, que es de $29,3 \mathrm{~cm}$, y $h$ es la altura del sensor respecto al eje horizontal $\overline{A C}$. Usando (2) se obtiene el radio de curvatura, $R C$, dado por:

$$
R C=\frac{1}{r}=\frac{8 h}{F^{2}+4 h^{2}}
$$

\section{ANÁLISIS EN COMPRESIÓN Y EN EXTENSIÓN}

\section{A. Mediciones y gráficas}

Las medidas de las tres FBGs por fibra arrojan resultados muy semejantes tanto en compresión como en extensión. También se realizaron mediciones en sentido inverso, es decir, desde una altura máxima hasta la posición donde la lámina está totalmente horizontal y no hay deformación. Este último procedimiento se realizó para evidenciar que no existen diferencias en las mediciones por causa de histéresis. Cada medición se llevó a cabo dos veces para detectar posibles cambios en la respuesta de longitud de onda.

En la fig. 5 se observan las dos mediciones para una FBG mientras se aumenta la altura y en condiciones de extensión. Los valores arrojados por la FBG ubicada por encima de la lámina, muestra un fenómeno de extensión y un desplazamiento de la longitud de onda hacia valores mayores

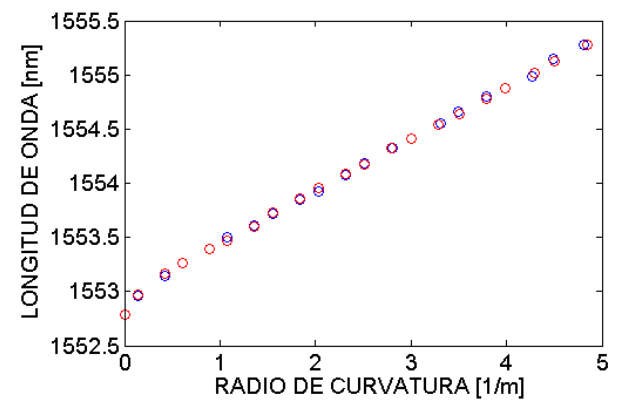

Fig. 5. Curva de longitud de onda vs Radio de curvatura en extensión.

[18].

En la fig. 6 se muestran los resultados para el proceso de compresión. En ambos casos se observa una tendencia lineal en los sensores de curvatura. Además la diferencia entre las dos pruebas, para una misma situación, es mínima .

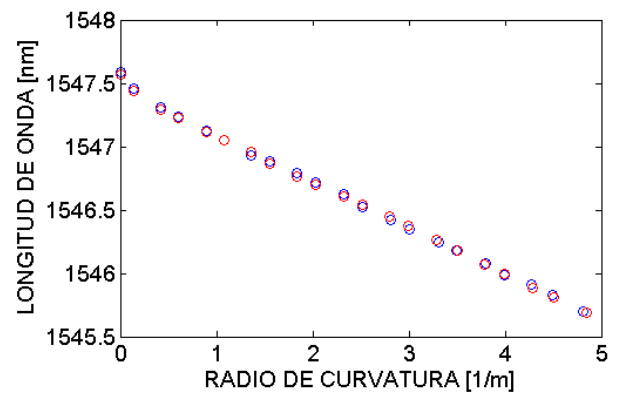

Fig. 6. Curva de longitud de onda vs Radio de curvatura en compresión.

La pendiente de la respuesta de la longitud de onda contra el radio de curvatura es igual para los tres sensores de cada fibra. La fig. 7 muestra la salida de los tres sensores para una fibra, la cual refleja la condición de compresión a medida que el radio de curvatura aumenta, como era de esperarse [19]. La única diferencia se encuentra a lo largo del eje de ordenadas, ya que se desea evitar el solape entre estas tres señales. Los cálculos se realizaron con valores diferenciales respecto a una condición inicial, para que tengan validez sobre los tres sensores de la fibra óptica y para que el resultado esperado sea indiferente de la posición de las FBGs a lo largo de la fibra.

\section{B. Cálculo de la distancia sensor-eje neutro}

Al momento de obtener las relaciones necesarias para determinar la distancia entre el sensor y el eje neutro se deben tener presentes las siguientes ecuaciones [20]:

$$
\begin{gathered}
\Delta \epsilon=\frac{\Delta L}{L} \\
\Delta \lambda=\frac{1,33 p m}{\mu \epsilon} \Delta \epsilon
\end{gathered}
$$




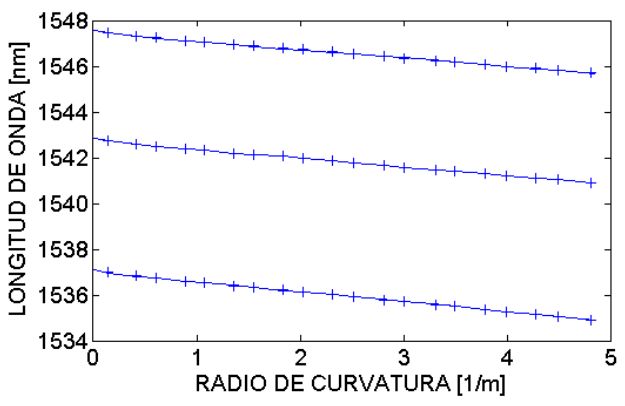

Fig. 7. Respuesta de tres FBGs de la fibra en compresión

La deformación que sufre la fibra está dada por (4), donde $\Delta L$ indica la variación de la longitud de la fibra, mientras que la relación entre la variación de longitud de onda, $\Delta \lambda$, y la variación de la deformación de la fibra, $\Delta \epsilon$, viene dada por (5), donde la lámina en posición horizontal tiene un $\Delta \epsilon=0$.

Así como la distancia sensor-eje neutro es constante a lo largo del experimento, al obtener una relación entre $\Delta \lambda$ y entre $\Delta R C$, el resultado tiende a ser una constante que es proporcional a la distancia sensor-eje neutro tanto en compresión como en extensión. De (4), referenciada a este experimento, $L$ tomará los valores del radio de la circunferencia y $\Delta L$ los valores de la distancia sensor-eje neutro. Combinando (4) y (5) junto con estos nuevos valores, se obtiene la distancia sensoreje neutro, $d$, dada por:

$$
d=\frac{\Delta \lambda}{\frac{1,33 p m}{\mu \epsilon} \Delta R C}
$$

Con (6), se puede determinar la variación entre la longitud de onda, $\Delta \lambda$, y la variación del radio de curvatura, $\Delta R C$, sin necesidad de calcular la variación de la deformación de la fibra, la variación en la longitud de la fibra o la fuerza aplicada a la estructura para deformarla. Si se conoce la distancia a la cual se ha adherido la fibra a la estructura respecto al eje neutro, se puede determinar el radio de curvatura a partir de la longitud de onda que refleje la FBG, ya que las variaciones son respecto a valores conocidos, que son obtenidos en condiciones iniciales.

\section{ANÁlisis dE RESUltados}

En (4),(5) y en (6), las variaciones de las variables están referidos respecto a un valor inicial, ya que son cambios en la longitud de onda, radio de curvatura y deformación, respectivamente. Para ello, se ha tomado como punto de referencia el momento en que la lámina se encuentra totalmente horizontal, y donde las FBGs reflejarán las longitudes de onda para las que fueron diseñadas originalmente y donde la fibra no sufre deformación.

Debido a la naturaleza de la lámina, luego de que queda bien tensionada tras una pequeña altura, el error en el cálculo de la distancia es mucho menor para posteriores alturas. En la Tabla I se observa el resultado de calcular la distancia, $d$, con (6) para diferentes alturas de la zona central de la lámina. Estas alturas están comprendidas entre $4,3 \mathrm{~cm}$ y $7,3 \mathrm{~cm}$, que
TABLE I

DISTANCIA SENSOR-EJE NEUTRO PARA DIFERENTES CURVATURAS

\begin{tabular}{|c|c|c|c|}
\hline $\begin{array}{c}\text { Compresión } \\
d[\mathrm{~mm}]\end{array}$ & $\begin{array}{c}\text { Extensión } \\
d[\mathrm{~mm}]\end{array}$ & $\begin{array}{c}\text { Desplazamiento } \\
\mathrm{x}[\mathrm{mm}]\end{array}$ & $\begin{array}{l}\text { Altura } \\
\mathrm{h}[\mathrm{cm}]\end{array}$ \\
\hline 0.3245 & 0.3293 & 4.31 & 4.3 \\
\hline 0.3211 & 0.3190 & 5.04 & 4.5 \\
\hline 0.3133 & 0.3187 & 6.38 & 4.8 \\
\hline 0.3177 & 0.3172 & 7.62 & 5 \\
\hline 0.3116 & 0.3145 & 9.15 & 5.3 \\
\hline 0.3106 & 0.3108 & 10.265 & 5.5 \\
\hline 0.3051 & 0.3078 & 12.5 & 5.8 \\
\hline 0.3020 & 0.3098 & 13.315 & 6 \\
\hline 0.2995 & 0.3087 & 15.39 & 6.3 \\
\hline 0.3009 & 0.3037 & 16.78 & 6.5 \\
\hline 0.2948 & 0.2984 & 18.44 & 6.8 \\
\hline 0.2946 & 0.3057 & 20.75 & 7 \\
\hline 0.2950 & 0.3013 & 23.78 & 7.3 \\
\hline
\end{tabular}

es un rango amplio de deformación de la lámina y de los sensores. El valor de $x$ en la Tabla I indica el desplazamiento horizontal de un extremo de la lámina.

El promedio de los valores de $d$ indica que, aproximadamente, la distancia sensor-eje neutro es de $0,31 \mathrm{~mm}$. Si se considera que la lámina es uniforme, y se suma la mitad del grosor de la lámina, el radio de la fibra y el espesor del pegamento usado para adherir la fibra a la lámina, se llega a concluir que el cálculo es una buena estimación de la distancia sensor-eje neutro, además el valor de la distancia, $d$, es muy aproximado en todas las mediciones.

Si cambia la distancia del sensor respecto al eje neutro, cambia también el valor de la longitud de onda para un mismo valor de altura o radio de curvatura. El efecto sobre este valor es un offset respecto al eje de las ordenadas de la fig. 5 y la fig. 6 .

Conocer la distancia sensor-eje neutro es útil, ya que conociendo solamente la longitud de onda no es posible determinar el radio de curvatura real. Con este cálculo de la distancia se logra mayor versatilidad en las mediciones de curvatura, y se evita el problema de tener que conocer de antemano la verdadera posición del sensor al momento de adherirlo a la superficie. Estos resultados serán válidos para alturas que no superen $\frac{L}{\Pi}$, aunque el interés del presente artículo giró alrededor de una altura máxima de $\frac{L}{4}$, donde $L$ es la longitud de la lámina.

\section{Conclusiones}

Se ha mostrado la utilidad de calcular el valor de distancia sensor-eje neutro mediante la relación de longitud de onda y radio de curvatura para procesos de compresión y extensión sobre una superficie. El valor de la distancia permite caracterizar de manera única el radio de curvatura, ya que con el valor de la distancia y el de la longitud de onda se puede determinar el radio de curvatura real. Indiferente del método de adhesión del sensor, el cual debería ser uniforme, y haciendo uso de (5) se obtiene el valor de la distancia del sensor al eje neutro, que es el eje donde la superficie idealmente no sufre compresión 
ni extensión.

Se ha logrado caracterizar un sensor de curvatura con un comportamiento lineal, el cual se analiza para deducir la relación de distancia del sensor-eje neutro para un rango de desplazamiento horizontal entre $4,31 \mathrm{~cm}$ y $23,78 \mathrm{~cm}$, o equivalentemente un rango de radio de curvatura entre $1,8 \mathrm{~m}^{-1}$ y $5 \mathrm{~m}^{-1}$ en una lámina de $29,3 \mathrm{~cm}$ de longitud. Es decir, el análisis fue realizado hasta una altura de $7,3 \mathrm{~cm}$. El sensor fabricado permitió manejar una respuesta lineal trabajando en tercera ventana, y permite desplazamientos de longitud de onda en compresión de hasta $2,22 \mathrm{~nm}$ y en extensión de hasta $2 \mathrm{~nm}$.

Los resultados obtenidos de este análisis están en sintonía con las dimensiones del sensor y muestran una buena repetitividad a lo largo de todas las mediciones.

\section{REFERENCES}

[1] W. Schulz and et al., "Advanced fiber grating strain sensor system for bridges, structures and highways," The Society of Photo-Optical Instrumentation Engineers, vol. 3325, pp. 212-221, 1998.

[2] M. Filograno and et al., "Estudio de deformaciones y vibraciones en una viga en ménsula mediante redes de bragg," in OPTOELO9 (Optoelectrónica 2009), Sexta Reunión Española de Optoelectrónica, 2009.

[3] R. García, Sistema de fabricación de altas prestaciones de redes de difracción de Bragg en fibra y aplicaciones al campo de los sensores ópticos y a los sistemas de comunicaciones ópticas. Universidad Politécnica de Valencia, 2008.

[4] J. Capmany and B. Ortega, Redes Ópticas. Universidad Politécnica de Valencia, 2006.

[5] L. Zhang and J. Qian, "Sensor net for shape detection of endoscope," in Proceedings of the IEEE (Institute of Electrical and Electronics Engineers), International Conference on Mechatronics \& Automation, 2005.

[6] A. Banerjee and et al., "Wavelength-division-multiplexed passive optical network (wdm-pon) technologies for broadband access," Optical Society of America, vol. 4 (11), pp. 737-758, 2005.

[7] M. Schaller and et al., "Smart cfrp systems for the controlled retrofitting of reinforced concrete members," The Society of Photo-Optical Instrumentation Engineer, vol. 7653, pp. C1-8, 2010.

[8] M. Mokhtar and et al., "Two-dimensional fibre grating packing design for simultaneous strain and temperature measurement," The Society of Photo-Optical Instrumentation Engineer, vol. 7653, pp. R1-3, 2010.

[9] N. Jaramillo, Mecánica Racional 10. Universidad de los Andes, n.d.

[10] N. Kim and N. Cho, "Estimation deflection of a simple beam model using fiber optic bragg-grating sensors," Society for Experimental Mechanics, vol. 1, pp. 433-439, 2004.

[11] G. Wehrle and et al., "Detecção da freqüência respiratória utilizando um sensor com redes de bragg em fibra Ótica," in CBEB (Congresso Brasileiro de Engenharia Biomédica), XVII Congresso Brasileiro de Engenharia Biomédica, 2000.

[12] R. Cazo and et al., "Sensor da deformação usando grades de bragg," Revista Científica Periódica, vol. 3 (2), pp. 76-79, 2000.

[13] N. Mohammand and et al., "Analysis and development of a tunable fiber bragg grating filter based on axial tension/compression," Journal of Lightware Technology, vol. 22 (8), pp. 2000-2013, 2004.

[14] X. Dong and et al., "Simultaneous displacement and temperature measurement with cantilever-based fiber bragg grating sensor," Elsevier, vol. 192, pp. 213-217., 2001.

[15] T. Imai and et al., "Dispersion tuning of a linearly chirped fiber bragg grating without a center wavelength shift by applying a strain gradient," IEEE Photonics technology Letters, vol. 10 (6), pp. 845-847, 1998.

[16] L. Jin and et al., "Two-dimensional bend sensing with a cantilevermounted fbg," Measurement Science and Technology, vol. 17, pp. 168172, 2005.

[17] O. Liévanos and et al., "Óptica oftálmica," Imagen Óptica, vol. 9, pp. 14-17, 2007.

[18] F. Araújo and et al., "Temperature and strain insensitive bending measurements with d-type fibre bragg grating," Measurement Science and Technology, vol. 12, pp. 829-833, 2001.
[19] X. Chen and et al., "Optical bend sensor for vector curvature measurement based on bragg grating in excentrix core polymer optical fibre," The Society of Photo-Optical Instrumentation Engineer, vol. 7503, pp. 1-4, 2009.

[20] Y. Yu and et al., "Bend sensor using an embedded etched fiber bragg grating," Microwave and Optical Technology Letters, vol. 43 (5), pp. 414-417, 2004

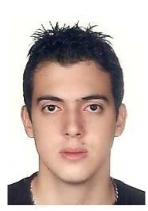

Luis Humberto Berrío was born in Medellín, Colombia, in 1987. He received the B.Sc. degree in Electronic Engineering from the Universidad de Antioquia (UdeA), Colombia, in 2011. He is Professor at the Departamento de Ingeniería Electrónica y Telecomunicaciones, Universidad de Antioquia. He is currently working towards the M. Sc. degree at Universidad Pontifica Bolivariana, Colombia, focusing on Automation and Control area. His current research interest are the Passive Optical Networks (PON) and the automation techniques.

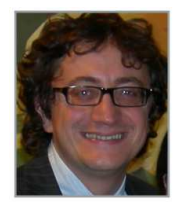

Salvador Sales is Professor at the Departamento de Comunicaciones, Universidad Politécnica de Valencia, SPAIN. He is also working in the ITEAM Research Institute. He received the M.Sc and the Ph.D. in Telecomunicación from the Universidad Politécnica de Valencia. He is co-author of more than 60 journal papers and 100 international conferences. He has been collaborating and leading some national and European research projects since 1997. His main research interests include optoelectronic signal processing for optronic and microwave systems, optical delay lines, fibre Bragg gratings, WDM and SCM ligthwave systems and semiconductor optical amplifiers.

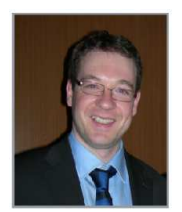

David Barrera was born in Valencia, Spain, in 1981. He received the M. Sc. degree in Telecommunications Engineering from the Universidad Politécnica de Valencia, UPV, in 2006. Since then, he has been working at the Optical and Quantum Communications Group of the ITEAM Research Institute. He is currently working towards the Ph.D. degree at Universidad Politécnica de Valencia and focusing on optical fibre sensing. His fields of interest are fibre Bragg gratings, optical fibre sensing and Polymer Optical Fibres. 